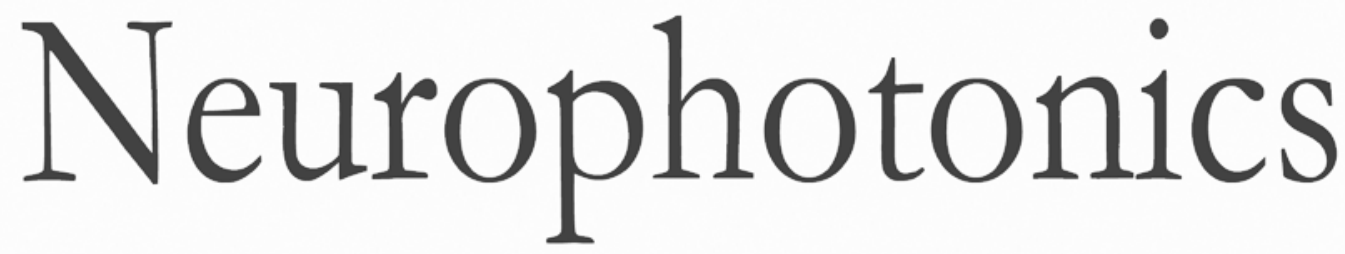

\title{
Erratum: Adaptive algorithm utilizing acceptance rate for eliminating noisy epochs in block-design functional near-infrared spectroscopy data: application to study in attention deficit/hyperactivity disorder children
}

Stephanie Sutoko

Yukifumi Monden

Tsukasa Funane

Tatsuya Tokuda

Takusige Katura

Hiroki Sato

Masako Nagashima

Masashi Kiguchi

Atsushi Maki

Takanori Yamagata

Ippeita Dand 


\title{
Erratum: Adaptive algorithm utilizing acceptance rate for eliminating noisy epochs in block-design functional near-infrared spectroscopy data: application to study in attention deficit/hyperactivity disorder children
}

\author{
Stephanie Sutoko, ${ }^{a}$ Yukifumi Monden, ${ }^{b, c}$ Tsukasa Funane, ${ }^{a, b}$ Tatsuya Tokuda, ${ }^{d}$ Takusige Katura, ${ }^{a}$ Hiroki Sato, ${ }^{a}$ \\ Masako Nagashima, ${ }^{\mathrm{b}}$ Masashi Kiguchi, ${ }^{a}$ Atsushi Maki, ${ }^{a}$ Takanori Yamagata, ${ }^{\mathrm{b}}$ and Ippeita Dand ${ }^{\mathrm{e}}$ \\ aHitachi Ltd., Research and Development Group, Center for Exploratory Research, Saitama, Japan \\ bichi Medical University, Department of Pediatrics, Shimotsuke, Japan \\ International University of Health and Welfare, Department of Pediatrics, Shiobara, Japan \\ ${ }^{\mathrm{d} C h u o ~ U n i v e r s i t y, ~ R e s e a r c h ~ a n d ~ D e v e l o p m e n t ~ I n i t i a t i v e s, ~ A p p l i e d ~ C o g n i t i v e ~ N e u r o s c i e n c e ~ L a b o r a t o r y, ~ T o k y o, ~ J a p a n ~}$ \\ e Jichi Medical University, Center for Development of Advanced Medical Technology, Shimotsuke, Japan
}

[DOI: 10.1117/1.NPh.5.4.049801]

This article [Neurophotonics 5(4), 045001 (Oct-Dec 2018)] was originally published online on 11 October 2018 with an error in Figure 13 on p. 13. The former figure included a mis-
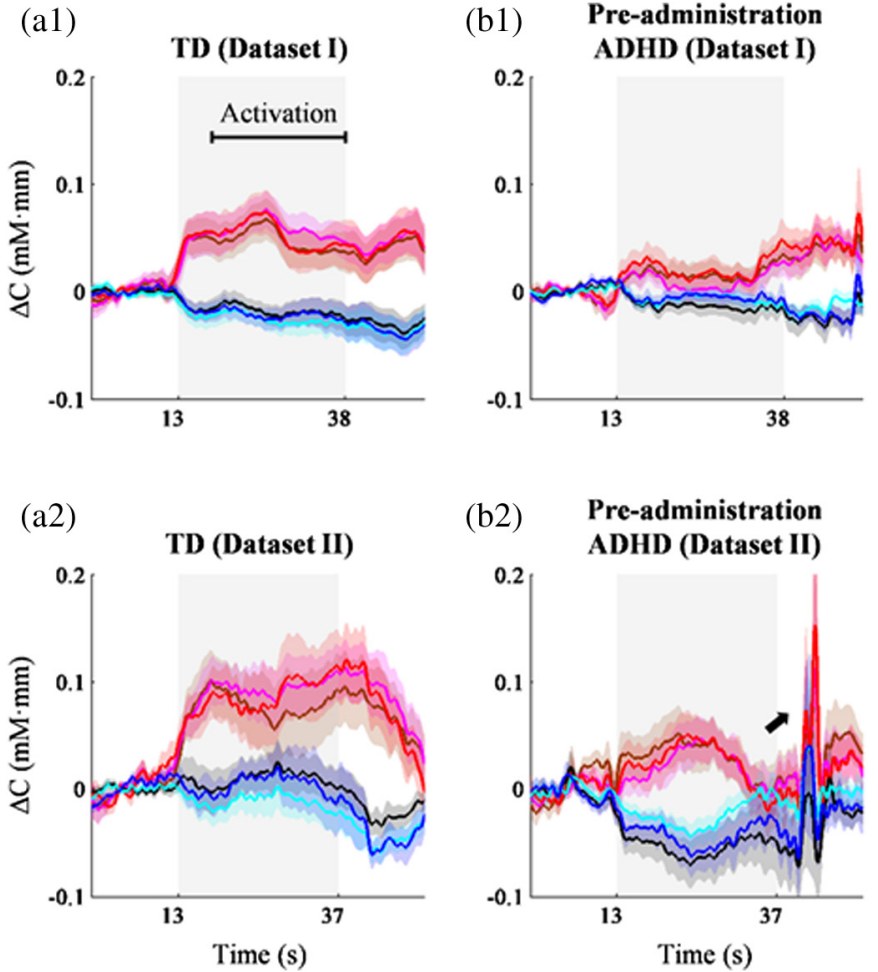
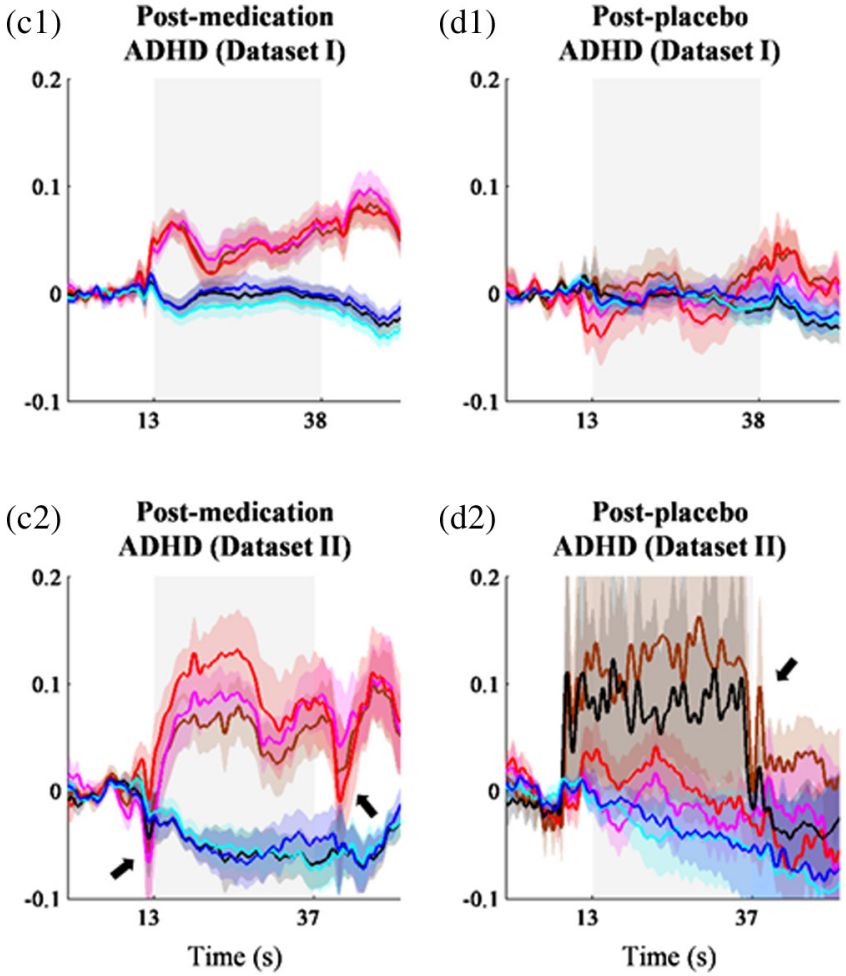

$(\mathrm{d} 2)$

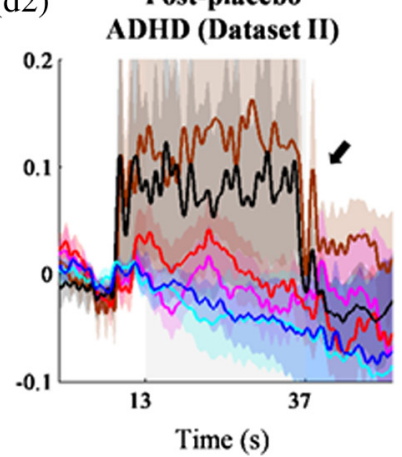

Fig. 13

This article was corrected online on 27 October 2018. It appears correctly in print. 